\title{
Targeted inhibition of mTORC2 prevents osteosarcoma cell migration and promotes apoptosis
}

\author{
XIAOKAI WANG ${ }^{1 *}$, PINGLIN LAI ${ }^{3 *}$, ZHONGMIN ZHANG $^{1}$, MINJUN HUANG $^{1}$, LIANG WANG $^{1}$, \\ MIN YIN $^{2}$, DADI JIN ${ }^{1}$, RONGPING ZHOU ${ }^{2}$ and XIAOCHUN BAI ${ }^{1,3}$ \\ ${ }^{1}$ Academy of Orthopedics of Guangdong Province, The Third Affiliated Hospital, Southern Medical University, Guangzhou, \\ Guangdong 510630; ${ }^{2}$ Department of Orthopedics, Second Affiliated Hospital of Nanchang University, \\ Nanchang, Jiangxi 330006; ${ }^{3}$ Department of Cell Biology, School of Basic Medical Science, \\ Southern Medical University, Guangzhou, Guangdong 510515, P.R. China
}

Received February 28, 2014; Accepted April 17, 2014

DOI: 10.3892/or.2014.3182

\begin{abstract}
Dysregulation of mammalian target of rapamycin (mTOR) signaling often occurs in many human malignant diseases, making it a potential target in the treatment of cancer. However, the effects of specifically targeted inhibition of mammalian target of rapamycin complex 2 (mTORC2) on osteosarcoma have not been reported. Three types of osteosarcoma cell lines (MG63/U2OS/Saos-2) were used in this study. Inhibition of mTORC2 was carried out by mTOR inhibitor PP242 and targeted siRNA. The anti-migration effect was evaluated through wound healing and Transwell assays. Osteosarcoma cells were either treated independently by inhibition of mTORC2 or in combination with cisplatin, and apoptosis was evaluated by staining with propidium iodide; PARP and caspase 7 expression levels were evaluated. Targeting of mTORC2 either by kinase inhibitor or rictor knockdown promoted cisplatin-induced apoptosis, but inhibition of mTORC1 either by rapamycin or raptor knockdown did not promote cisplatin-induced apoptosis. Furthermore, inhibition of mTORC2 but not mTORC1 effectively prevented osteosarcoma cell migration. These results suggest that agents that inhibit mTORC2 have advantages over mTORC1 inhibitors in the treatment of osteosarcoma. The present study provides a strong rationale for testing the use of mTORC1/2 inhibitors or the combination of mTORC $1 / 2$ inhibitors and cisplatin in the treatment of osteosarcoma.
\end{abstract}

Correspondence to: Dr Rongping Zhou, Department of Orthopedics, Second Affiliated Hospital of Nanchang University, Nanchang, Jiangxi 330006, P.R. China

E-mail: rong8618241@126.com

Dr Xiaochun Bai, Department of Cell Biology, School of Basic Medical Science, Southern Medical University, 1838 North Guangzhou Avenue, Guangzhou, Guangdong 510515, P.R. China

E-mail: baixc15@smu.edu.cn

${ }^{*}$ Contributed equally

Key words: osteosarcoma, mammalian target of rapamycin complex 2, mTOR kinase inhibitor, apoptosis, migration

\section{Introduction}

Osteosarcoma is the most common primary malignant neoplasm of bone and is associated with rapid progression and poor prognosis. Surgical amputation can achieve local tumor elimination, yet $80 \%$ of osteosarcoma patients have a low 2-year survival rate due to distant metastasis, particularly for patients receiving single surgical treatment (1-3). Consequently, lesionectomy combined with assistant post/preoperative chemotherapy has been considered significant and necessary (4). Currently, chemotherapeutic drugs include cisplatin, epirubicin, etopside, methotrexate and cyclophosphamide (3). These drugs are known to cause serious systemic toxicity, when used either as a single agent or in combination with other drugs. Therefore, there is an urgent need to develop additional available chemotherapeutic strategies or seek safer and more effective chemotherapeutic agents for the treatment of osteosarcoma.

The mammalian target of rapamycin (mTOR) is a serine/threonine kinase at the nexus between oncogenic phosphoinositide 3-kinase (PI3K)/Akt signaling and critical downstream pathways that drive cancer cell growth, survival and resistance to therapeutic agents $(5,6)$. The functions of mTOR are elicited by the context of two multiprotein complexes termed mTOR complex 1 (mTORC1) and mTORC2. mTORC1 is sensitive to rapamycin while mTORC2 is insensitive to acute rapamycin treatment. The major downstream effectors of mTORC1 are the ribosomal subunit S6 kinase 1 (S6K1) and the eukaryotic initiation factor $4 \mathrm{E}$ binding protein 1 (4E-BP1), two regulators of protein translation initiation and cell growth. mTORC2 phosphorylates and activates protein kinase B/Akt (Ser473), an important pro-survival kinase in cells. Consistent with its role as a growth-promoting pathway, mTOR signaling is dysregulated in $>50 \%$ of all human cancers including osteosarcoma and is a major cancer drug target (7).

As first-generation mTOR inhibitors, rapamycin and rapalogs (everolimus, temsirolimus) can slow the proliferation of cancer cell lines and have achieved some success in cancer treatment (8). Unfortunately, their overall efficacy as cancer therapeutics has been limited to a few rare cancers, including mantle cell lymphoma, renal cell carcinoma and endometrial cancer $(7,9)$. 
Clinical trials have shown that osteosarcoma patients are not sensitive to rapalogs when employed in a monotherapy setting.

Second-generation mTOR ATP competitive inhibitors have been recently developed and are able to completely suppress both mTORC1/C2 complex-mediated signaling, thereby suppressing the feedback activation of Akt (10-15). Importantly, they have shown marked improvement in antitumor activity in vivo and in vitro, and the effectiveness of these drugs in cancer treatment is currently being tested in clinical trials $(7,16,17)$. The effects of mTOR kinase inhibitors on osteosarcoma, however, have not been reported. Furthermore, mTORC2 is emerging as a promising therapeutic target as its activity is essential for the transformation and vitality of various types of cancer cells. Consequently, it is important to determine the efficacy of targeting mTORC2 in osteosarcoma. In the present study, we compared the inhibitory effects of the targeting of mTORC1 with mTORC2 on a variety of osteosarcoma cell lines and demonstrated that targeted inhibition of mTORC2, but not mTORC1, prevents osteosarcoma cell migration and promotes cell apoptosis.

\section{Materials and methods}

Reagents and antibodies. PP242 was purchased from Active Biochemicals. Co. (Hong Kong); rapamycin was from Sigma Chemical Co. (St. Louis, MO, USA). PP242 and rapamycin were diluted in dimethyl sulfoxide (DMSO). The following antibodies were used: phospho-S6 (S235/236), raptor, cleaved poly(ADP-ribose) polymerase (PARP) and caspase 7 from Cell Signaling Technology, Inc. (Beverly, MA, USA); S6, actin, P-Akt (S473), mTOR, rictor and Akt were from Santa Cruz Biotechnology, Inc. (Santa Cruz, CA, USA).

Cell lines. Osteosarcoma cell lines, MG63, U2-OS and Saos-2, were purchased from the Type Culture Collection of the Chinese Academy of Sciences (Shanghai, China). MG63 cells were grown in Dulbecco's modified Eagle's medium (DMEM) with $10 \%$ fetal bovine serum (FBS), and U2-OS and Saos-2 cells were grown in McCoy's 5A medium with $10 \%$ FBS. All media and FBS were from Gibco-BRL (Rockville, MD, USA). Cells were cultured at $37^{\circ} \mathrm{C}$ in a humidified atmosphere consisting of $5 \% \mathrm{CO}_{2}$ and $95 \%$ air.

Western blotting. Western blotting was performed as previously described $(18,19)$. In brief, cells were lysed in a buffer containing $1 \%$ SDS. Equal amounts of whole protein extract were resolved on SDS-polyacrylamide gel, transferred to a nitrocellulose membrane (Amersham Biosciences, Italy), probed overnight at $4^{\circ} \mathrm{C}$ with antibodies and then revealed using the ECL western blot analysis system.

Drug interaction analysis. Drug combination analysis was performed as previously described (19). In briefly, the method describe by Chou and Talalay (20), and multiple drug doseeffect calculations and the combination index plots were generated using CalcuSyn 2.1 software (Biosoft, Cambridge, UK). We assessed the effects of drug interactions by combination index $(\mathrm{CI})$ values. $\mathrm{CI}<1$ indicated synergism, whereas $\mathrm{CI}=1$ and $\mathrm{CI}>1$ indicated additive effect and antagonism, respectively. The pro-apoptosis studies were performed by using 31, 62, 125, 250 and $500 \mathrm{nM}$ of PP242 and rapamycin; both studies were combined with 31, 62, 125, 250 and $500 \mathrm{nM}$ cisplatin treatment.

RNA interference. Human mTOR-, raptor- and rictor-specific siRNAs were chemically synthesized by GenePharma Co., Ltd. (Shanghai, China). Target sequences of these siRNAs were mTOR (50-GAGCCUUGUUGAUCCUUAA-30), raptor (50-CGAGAUUGGACGACCAAAU-30) and rictor (50-GACUAUCCAUAAUCCUUA-30). Cells were transfected with the siRNAs at $60 \%$ confluency using Lipofectamine 2000 (Invitrogen, Grand Island, NY, USA) according to the manufacturer's instructions.

Cell migration and motility analysis. Migration ability was assessed by a wound healing assay. Cells were seeded in 12-well plates and treated with PP242 (200 nM) or rapamycin (200 nM) or transfected with the negative control (NC), mTOR, raptor or rictor siRNA, and grew until reaching $100 \%$ confluency. Cells were then treated with $2 \mu \mathrm{g} / \mathrm{ml}$ of mitomycin $\mathrm{C}$ for $24 \mathrm{~h}$ in a minimum serum medium (containing $0.5 \% \mathrm{FBS}$ ). After using a pipette tip to create a scratch in the confluent cells, the wound distance was visualized at regular intervals of time (24/48 h) at x100 magnifications using an inverted microscope (Nikon Diaphot). The cell motility assay was performed on a Transwell plate. Cells $\left(1.0 \times 10^{5}\right)$ were seeded in a Matrigel-coated chamber (BD Biosciences). Cells were seeded in serum-free media and translocated toward complete growth media. After $12 \mathrm{~h}$ of incubation with PP242 (200 nM) and rapamycin $(200 \mathrm{nM})$ at $37^{\circ} \mathrm{C}$, invaded cell were fixed and stained in dye solution containing $20 \%$ methanol and $0.1 \%$ crystal violet. The cells which had migrated or invaded were imaged using a BH-2 inverted microscope (Olympus).

Apoptotic cell staining. Cells were treated with PP242 $(500 \mathrm{nM})$ and rapamycin $(500 \mathrm{nM})$ or transfected with siRNA in medium without serum for $36 \mathrm{~h}$. Apoptotic cells were assessed using a PI assay kit (MultiSciences Biotech Co. Ltd., Beijing, China) according to the manufacturer's instructions. Briefly, the treated cells were carefully washed three times with PBS, and then staining was performed with a mixture containing $10 \mathrm{mg} / \mathrm{ml} \mathrm{PI}$ and PBS in the wells and stained for $15 \mathrm{~min}$ at $37^{\circ} \mathrm{C}$ in the dark. Photomicrographs of the stained cells were then recorded under a fluorescence microscope with digital equipment, and the apoptotic cells and viable cells were counted visually. The apoptosis ratio $=$ apoptotic cells $/$ total cells x $100 \%$.

Statistical analysis. Data analysis was performed with SPSS 13.0. Statistical analysis was performed by applying one-way analysis of variance (ANOVA). Data are presented as means \pm standard deviation (SD), and $\mathrm{p}<0.05$ was considered to indicate statistical significance.

\section{Results}

mTOR downstream kinases are deregulated by mTORC1 inhibitor rapamycin and $m$ TORC1/2 inhibitor PP242. We first examined the distinct effects of the targeting of mTORC1 by rapamycin and the targeting of $\mathrm{mTORC} 1 / 2$ by mTOR kinase 

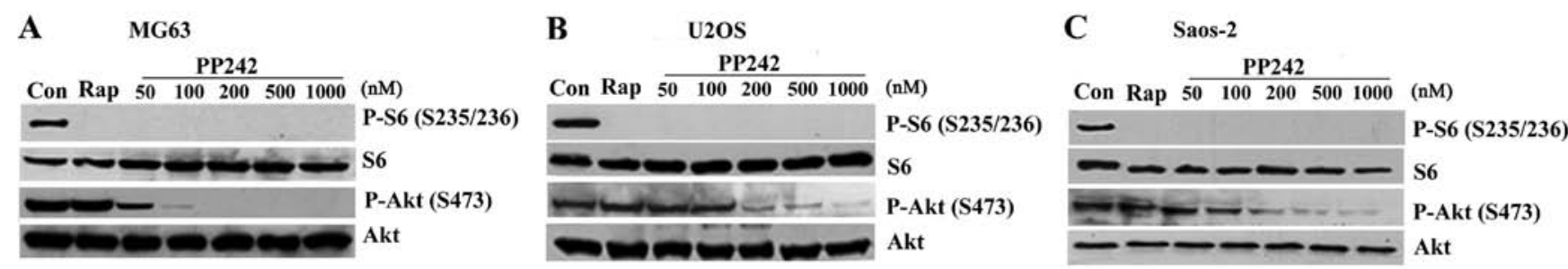

Figure 1. Targeted inhibition of mTORC1/2 signaling by mTOR kinase inhibitor PP242 in osteosarcoma cells. (A) MG63, (B) U2OS and (C) Saos-2 cells were incubated with $50 \mathrm{nM}$ rapamycin or the indicated doses of PP242 for $30 \mathrm{~min}$. Cell lysates were then subjected to immunoblotting for levels of phospho-Akt (S473), Akt, phospho-S6 (S235/236) and S6. Rap, rapamycin; mTORC1, mammalian target of rapamycin complex 1.

A

$\mathrm{h}$

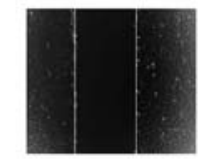

$24 \mathrm{~h}$

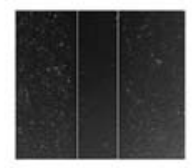

$48 \mathrm{~h}$

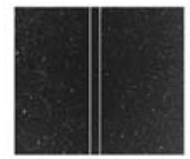

$\mathrm{C}$
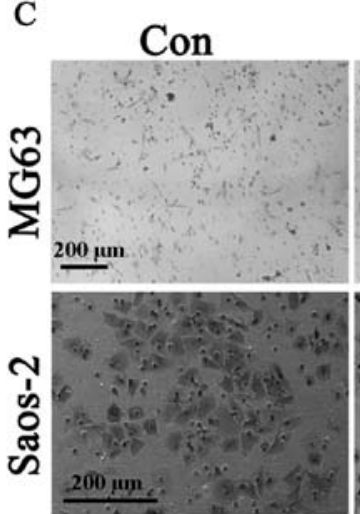

Rapamycin
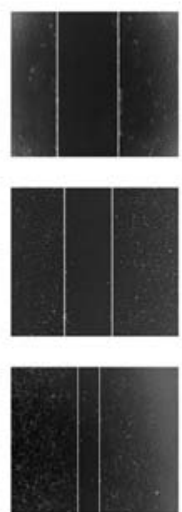

Rap

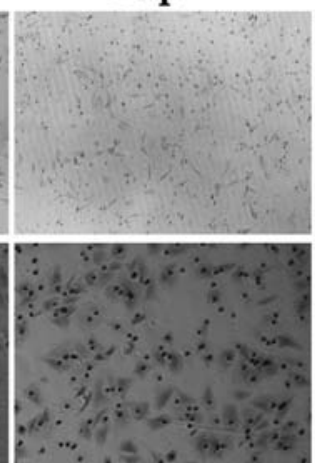

PP242
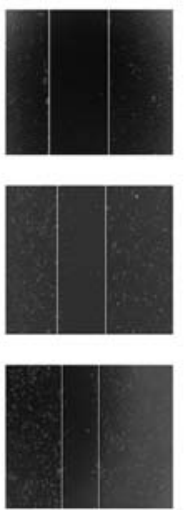

PP242

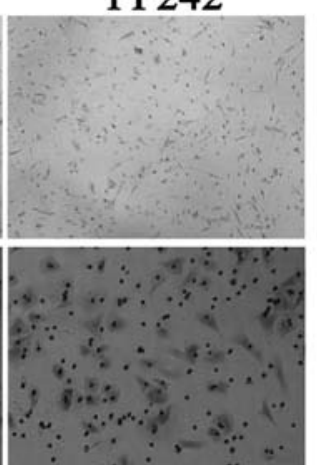

B

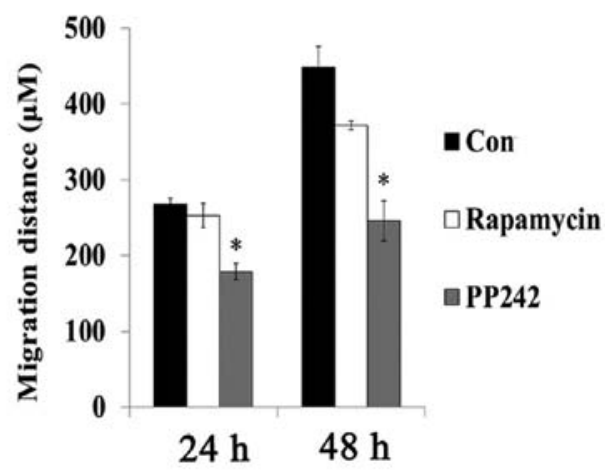

D

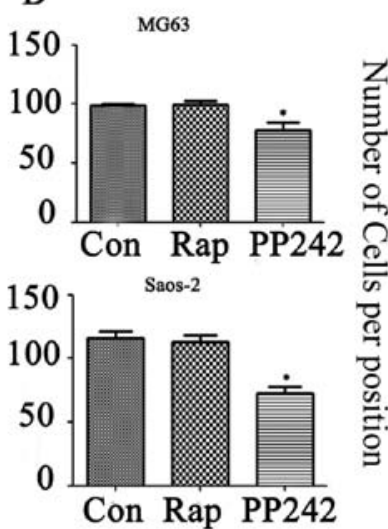

Figure 2. mTORC1/2 kinase inhibitor PP242 prevents osteosarcoma cell migration. (A) Saos-2 cells were treated with 100 nM of rapamycin or PP242 for $30 \mathrm{~min}$, and subsequently subjected to wound healing assay as described in Materials and methods. (B) The wound distances were measured under a light microscope. * $\mathrm{p}<0.01$ compared with control and rapamycin treatment. (C) Transwell assay was performed on MG63 and Saos-2 cells treated with rapamycin $(100 \mathrm{nM})$ or PP242 (100 nM). (D) The numbers of cells that migrated to the bottom side of the membrane were calculated. Data are presented as means \pm SE of the migrated cells. * p $<0.05$ statistically significant difference between PP242 and control. Rap, rapamycin; mTORC1, mammalian target of rapamycin complex 1 .

inhibitor PP242 on their downstream signals in osteosarcoma MG63, U2OS and Saos-2 cell lines. Although these two drugs were able to effectively suppress phosphorylation of S6 (S235/236) in all tested cell lines, only PP242 dose dependently (50-1000 nM) inhibited phosphorylation of Akt (S473), an mTORC2 phosphorylation site. Rapamycin treatment did not cause any significant changes in the phosphorylation level of Akt (S473) (Fig. 1A-C). This indicated that mTOR kinase inhibitors profoundly diminished both mTORC1 and mTORC2 signaling, whereas rapamycin only suppressed mTORC1 in the osteosarcoma cell lines.
Inhibition of mTORC1/2 or mTORC2 prevents osteosarcoma cell migration. Metastasis is the major cause of mortality and morbidity of osteosarcoma patients. Invasion of cancer cells into surrounding tissue and vasculature is an initial step in tumor metastasis. This requires migration of cancer cells (21). The effects of targeted inhibition of mTORC1 and/or mTORC2 on cell migration were examined by wound healing assay. PP242treated Saos- 2 cells exhibited a slower migration speed than the control and rapamycin-treated Saos-2 cells (Fig. 2A and B). In addition, the Transwell assay was performed to confirm the inhibitory effect of PP242 on osteosarcoma cell motility. As 
A

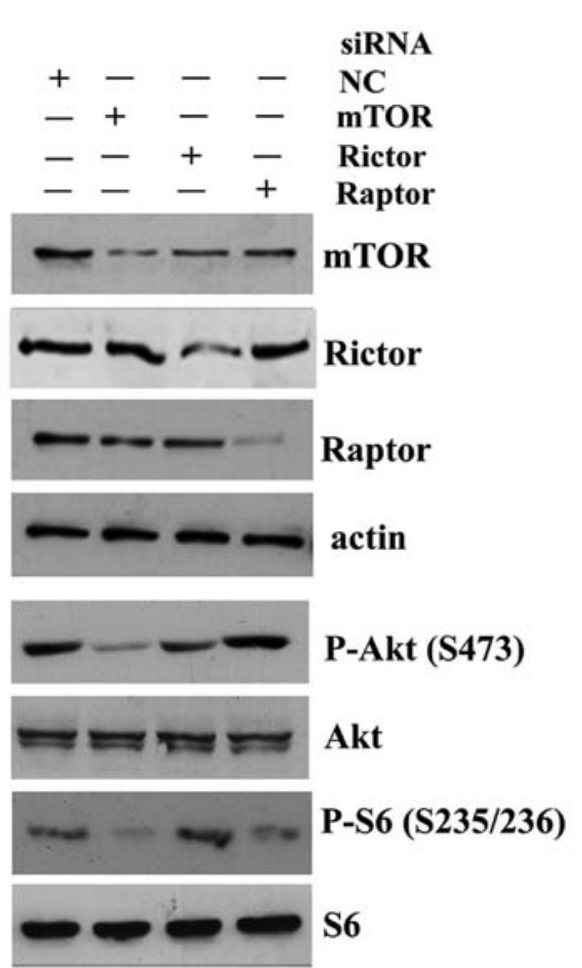

B

$0 \mathrm{~h}$

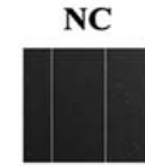

$48 \mathrm{~h}$

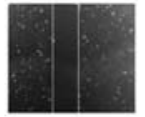

C

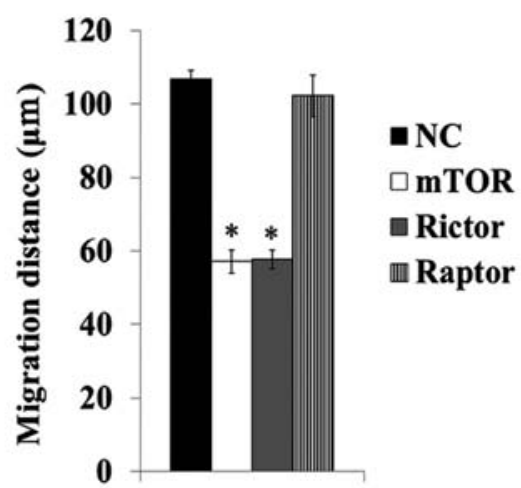

Figure 3. Targeted inhibition of mTORC2 prevents osteosarcoma cell migration. (A) Saos-2 cells were transfected with negative control (NC), mTOR, raptor or rictor siRNA for $48 \mathrm{~h}$, and cell lysates were subjected to immunoblotting for levels of mTOR, raptor, rictor, phospho-Akt (S473), Akt, phospho-S6 (S235/236) and S6. (B) Saos-2 cells were transfected with NC, mTOR, raptor, or rictor siRNA for $48 \mathrm{~h}$, and subsequently subjected to a wound healing assay as described in Materials and methods. (C) The wound distances were measured under a light microscope. "p $<0.01$ compared with NC and raptor siRNA treatment. mTORC2, mammalian target of rapamycin complex 2 .

A

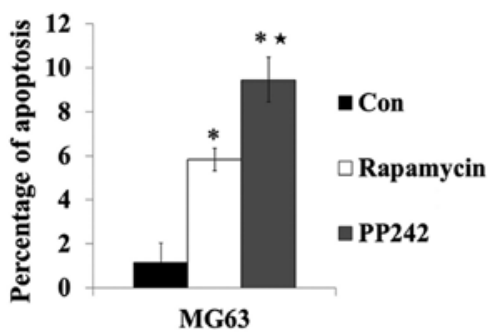

B

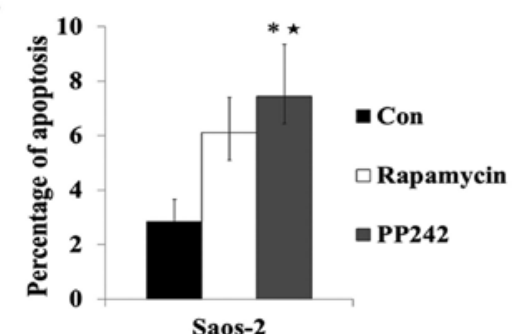

C

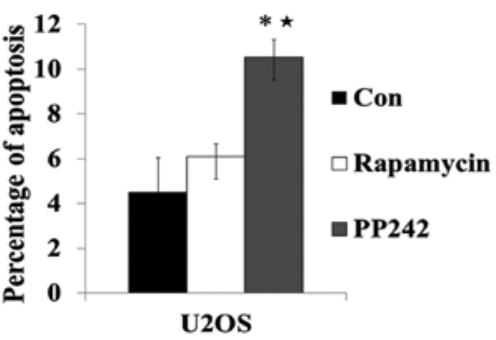

MG63

Con Rap PP242

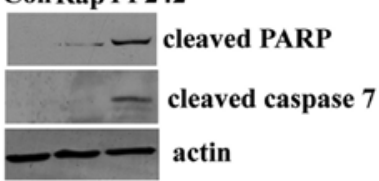

Saos-2
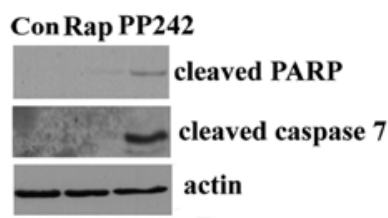

U2OS

Con Rap PP242

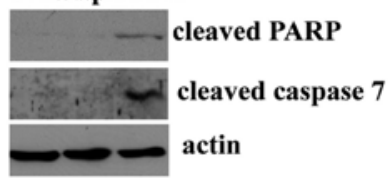

Figure 4. PP242 is more effective than rapamycin in promoting apoptosis in osteosarcoma cells. (A) MG63, (B) Saos-2 and (C) U2OS cells were incubated with $100 \mathrm{nM}$ rapamycin or $100 \mathrm{nM}$ of PP242 for $36 \mathrm{~h}$ in serum-free medium. Apoptotic cell death was then quantified using PI staining. Lysates from cells treated with $100 \mathrm{nM}$ of rapamycin or PP242 for $36 \mathrm{~h}$ were subjected to immunoblotting for levels of cleaved PARP, caspase 7 and actin. ${ }^{\star} \mathrm{p}<0.01 \mathrm{compared}$ with control and rapamycin treatment; ${ }^{*} \mathrm{p}<0.01$ compared with control and rapamycin. 


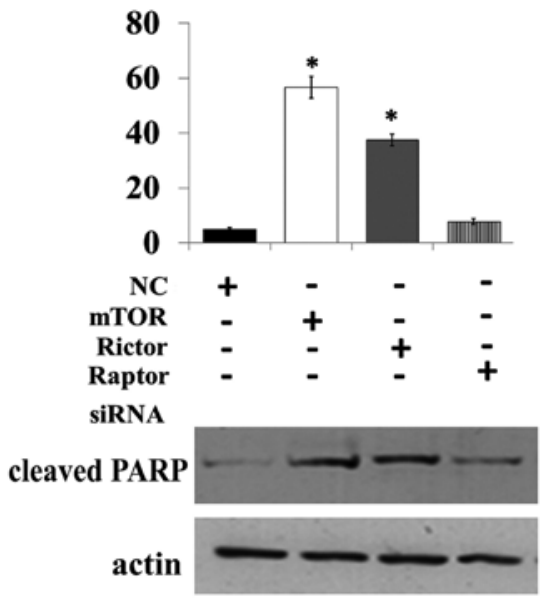

Figure 5. Rictor and mTOR knockdown, but not raptor knockdown potentiates apoptosis in osteosarcoma cells. Saos- 2 cells were transfected with negative control (NC), mTOR, raptor or rictor siRNA for $48 \mathrm{~h}$, and subsequently serum-starved for another $36 \mathrm{~h}$. Apoptotic cell death was then quantified using PI staining. Cell lysates were subjected to immunoblotting for levels of cleaved PARP and actin. "p $<0.01$ compared with NC and raptor siRNA treatment. mTOR, mammalian target of rapamycin.

expected, the number of migrated cells markedly decreased in the PP242-treated but not in the rapamycin-treated MG63 and Saos-2 cells $(\mathrm{p}<0.05)$ (Fig. $2 \mathrm{C}$ and $\mathrm{D})$. These results indicated that the inhibitor of mTORC1/2 prevented osteosarcoma cell migration. To further identify the roles of mTORC1 and mTORC2 in osteosarcoma cell migration, the effects of raptor, mTOR or rictor knockdown on migration were examined. Raptor, rictor or mTOR siRNA markedly decreased the protein levels of raptor, rictor or mTOR and reduced the phosphorylation of their outputs S6 (S235/236) and Akt (S473), respectively in Saos-2 cells (Fig. 3A). Knockdown of rictor and mTOR, but not raptor significantly repressed cell migration $(p<0.01)$ (Fig. 3B and C). These results further confirmed the critical role of mTORC2 in osteosarcoma cell migration.

Inhibition of mTORC2 but not mTORC1 promotes apoptosis in osteosarcoma cells. Akt represents an important intracellular survival signaling under a variety of conditions (22). Rapamycin did not inhibit mTORC2/Akt, and accordingly it did not promote apoptosis in osteosarcoma MG63, U2OS and Saos-2 cells (Fig. 4A-C). Consistent with the inhibitory activities of PP242 on mTORC2/Akt (S473) phosphorylation (Fig. 1), the drug significantly enhanced cleavage of PARP, caspase 7 and the number of apoptotic cells in the serum-starved MG63, U2OS and Saos-2 cells (Fig. 4A-C).

To further identify the different role of mTORC1 and mTORC2 in osteosarcoma cell apoptosis, the raptor, rictor or mTOR siRNA was transfected into Saos- 2 cells. The results showed that knockdown of mTOR and rictor but not raptor were able to increase the number of apoptotic cells and the level of cleaved-PARP in the Saos-2 cells (Fig. 5). Hence, we demonstrated that targeting of mTORC2 but not mTORC1 promoted apoptosis in the osteosarcoma cells.

Targeting of mTORC2 but not mTORC1 promotes cisplatininduced apoptosis in osteosarcoma cells. Cisplatin is a common chemotherapeutic agent that induces apoptosis in a
A

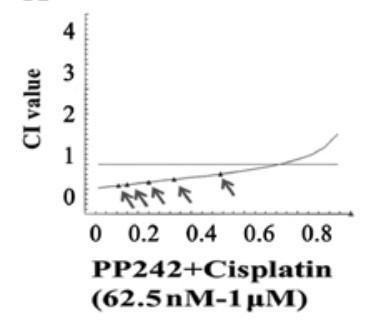

U2OS

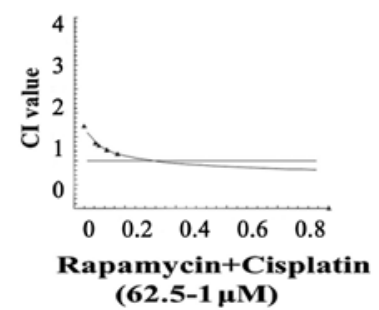

MG63

B
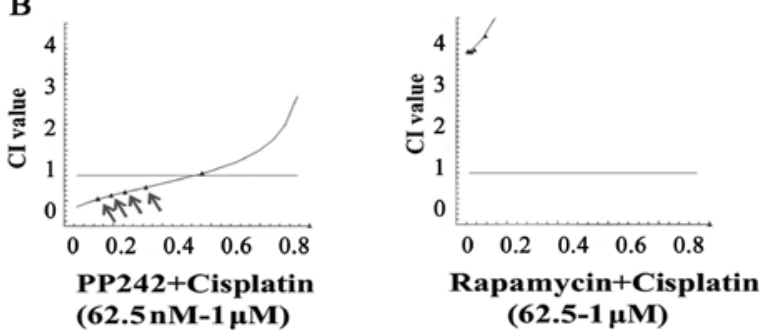

C
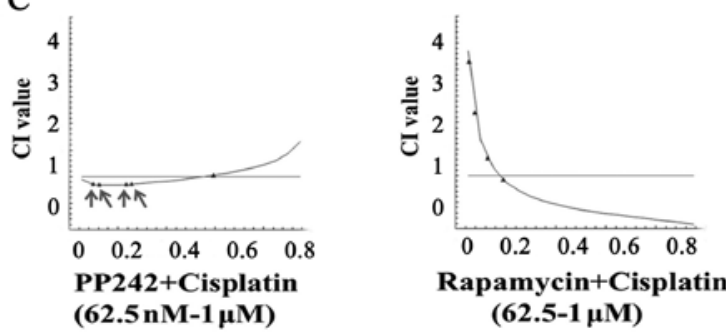

Figure 6. PP242 but not rapamycin exhibits synergistic interactions with cisplatin in promoting cell apoptosis. (A) U2OS, (B) MG63 and (C) Saos-2 cells were treated with different concentrations of PP242 or rapamycin (31, $62,125,250$ and $500 \mathrm{nM})$ and cisplatin $(31,62,125,250$ and $500 \mathrm{nM})$ for $36 \mathrm{~h}$, and apoptotic cell death was then quantified using PI staining. CI values were calculated with the CalcuSyn software.

variety of cancer cell lines including osteosarcoma cells (23). Drug CI analysis is a generalized method for analyzing the effects of multiple drugs and for determining summation, synergism and antagonism.

We used the drug CI analysis to examine the interaction of mTORC1 inhibitor rapamycin or mTORC1/2 inhibitor PP242 with cisplatin. We performed the dose-response studies in osteosarcoma cell lines to obtain the apoptotic rate, and we calculated the CI values by the CalcuSyn program. When PP242 was applied at most concentrations in all tested cell lines, the CI values were $<1$, indicating synergistic proapoptotic interactions between PP242 and cisplatin (Fig. 6). In contrast, rapamycin did not show any synergistic interactions with cisplatin, as the CI values were $>1$ (Fig. 6). Most importantly, rictor or mTOR knockdown, but not raptor knockdown, markedly enhanced cisplatin-induced cleavage of PARP and the number of apoptotic cells in Saos-2 cells (Fig. 7). Taken together, our results indicate that targeted inhibition of mTORC 2 but not mTORC1 markedly enhanced cisplatininduced apoptosis in osteosarcoma cells.

\section{Discussion}

In the present study, we demonstrated that either inhibition of mTORC1/2 by PP242 or knockdown of mTOR or rictor by 


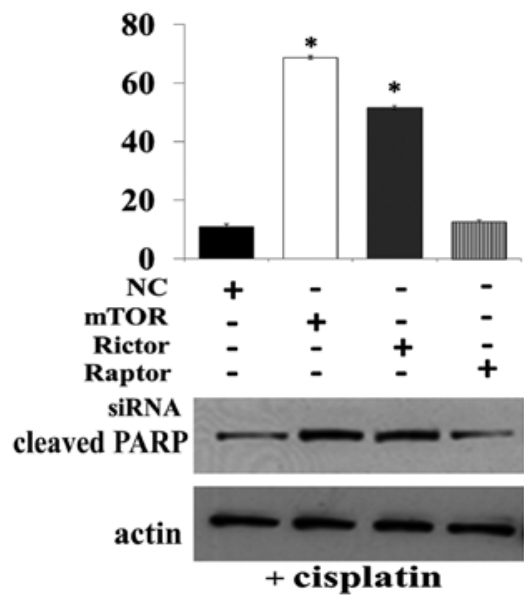

Figure 7. Rictor and mTOR knockdown, but not raptor knockdown potentiates cisplatin-induced osteosarcoma cell apoptosis. Saos-2 cells were transfected with negative control (NC), mTOR, raptor or rictor siRNA for $48 \mathrm{~h}$, and subsequently serum-starved for another $36 \mathrm{~h}$, and then treated with $200 \mathrm{nM}$ of cisplatin for another $36 \mathrm{~h}$. Apoptotic cell death was then quantified using PI staining. Cell lysates were subjected to immunoblotting for levels of cleaved PARP and actin. " p $<0.01$ compared with $\mathrm{NC}$ and raptor siRNA treatment. mTOR, mammalian target of rapamycin.

siRNA prevents migration and promotes apoptosis in osteosarcoma cell lines. We also demonstrated the synergistic antitumor activity achieved by combining mTOR kinase inhibitor PP242 with cisplatin. Our findings suggest that agents that inhibit either mTORC2 or mTORC1/2 may have advantages over selective mTORC1 inhibitors in the treatment of osteosarcoma. Given that mTOR kinase inhibitors (e.g., OSI-027) are undergoing clinical trials, this study provides a strong rationale for testing the use of mTOR kinase inhibitors or the combination of mTOR kinase inhibitors with traditional chemotherapeutic drug cisplatin for the treatment of osteosarcoma.

It has been well established that mTOR signaling plays key roles in the pathogenesis and progression of osteosarcoma and is a major cancer drug target. However, clinical trials have shown that osteosarcoma patients are not sensitive to rapalogs when employed in a monotherapy setting. The known mechanisms for rapalog resistance are as follows. i) S6K is exquisitely inhibited, yet the control of $4 \mathrm{E}-\mathrm{BP} 1$ and $\mathrm{mRNA}$ translation is not sensitive (24); ii) mTORC2 activity is not acutely blocked and iii) there is a feedback loop between mTORC1 and Akt. Treatment with rapalogs results in elevated Akt activity, which serves as a mechanism to enhance cell survival when mTORC1 is inhibited (25).

In the past decade, much research has focused on the antitumor effect of second-generation mTOR kinase inhibitors. This research has not only furthered the understanding of the mTOR signaling network, but has also contributed to to the development of many mTORC1/2 inhibitors including Torin1, PP242, PP300 and OSI-027. The antitumor effects of these mTOR inhibitors have been assessed in many types of cancers (26). Some of the results demonstrated high effectiveness, resulting in the move to clinical trials. Nevertheless, the mechanism involved in the mTOR kinase regulation of cancer cells and the specific target of mTOR inhibitors remain unclear. Their effects on osteosarcoma, and how the therapeutic function is regulated require further research. In the present study, we compared the antitumor activity of the targeted inhibition of mTORC1 and mTORC2 in osteosarcoma. Our results demonstrated that although rapamycin or PP242 suppressed proliferation in a variety of osteosarcoma cell lines, inhibition of mTORC 2 by PP242 or rictor knockdown effectively prevented osteosarcoma cell migration and promoted cell apoptosis, while inhibition of mTORC1 by rapamycin or raptor knockdown neither prevented osteosarcoma cell migration nor promoted cell apoptosis. These data suggest that mTOR kinase inhibitors are more effective than rapalogs to suppress osteosarcoma and that promotion of apoptosis and suppression of cell migration may contribute to the therapeutic effects of mTORC $1 / 2$ inhibitors.

Recent studies have suggested that mTORC2 activity is essential for the transformation and vitality of a number of cancers driven by mutations of PI3K or loss of PTEN, which include glioma and prostate cancers $(27,28)$. Yet, the roles of mTORC2 in carcinogenesis and progression of osteosarcoma are not known. Although both mTORC1 and mTORC2 have been reported to mediate epithelial-mesenchymal transition (EMT) and cell motility in epithelial, colon cancer and podocytes (29-31), we found that inhibition of mTORC2 but not mTORC1 promoted apoptosis and suppressed migration in osteosarcoma cells, indicating the critical role of mTORC2 in osteosarcoma cell survival and migration. Thus, mTORC2 is a promising therapeutic target in the prevention and treatment of osteosarcoma. mTOR kinase inhibitors may prevent mTORC2 activity. However, concurrent inhibition of mTORC1 may introduce hyperactivation of PI3K signaling and possible deleterious effects to normal host tissues, which limits their therapeutic potential (32). Thus, mTORC2-specific inhibitors may be promising therapeutic agents for osteosarcoma.

\section{Acknowledgements}

This study was supported by the National Natural Sciences Foundation of China (31271271, 31000633 and 81260401), and GDUPS (2011).

\section{References}

1. Biermann JS, Adkins D, Benjamin R, et al: Bone cancer. J Natl Compr Cancer Netw 5: 420-437, 2007.

2. Demetri GD, Baker LH, Benjamin RS, et al: Soft tissue sarcoma. J Natl Compr Cancer Netw 5: 364-399, 2007.

3. Marina N, Gebhardt M, Teot L and Gorlick R: Biology and therapeutic advances for pediatric osteosarcoma. Oncologist 9: 422-441, 2004.

4. Patel SR, Vadhan-Raj S, Burgess MA, et al: Results of two consecutive trials of dose-intensive chemotherapy with doxorubicin and ifosfamide in patients with sarcomas. Am J Clin Oncol 21: 317-321, 1998.

5. Zoncu R, Efeyan A and Sabatini DM: mTOR: from growth signal integration to cancer, diabetes and ageing. Nat Rev Mol Cell Biol 12: 21-35, 2011.

6. Guertin DA and Sabatini DM: An expanding role for mTOR in cancer. Trends Mol Med 11: 353-361, 2005.

7. Fasolo A and Sessa C: Targeting mTOR pathways in human malignancies. Curr Pharm Des 18: 2766-2777, 2012.

8. Benjamin D, Colombi M, Moroni C and Hall MN: Rapamycin passes the torch: a new generation of mTOR inhibitors. Nat Rev Drug Discov 10: 868-880, 2011.

9. Houghton PJ: Everolimus. Clin Cancer Res 16: 1368-1372, 2010.

10. Guertin DA and Sabatini DM: The pharmacology of mTOR inhibition. Sci Signal 2: pe24, 2009. 
11. Feldman ME, Apsel B, Uotila A, et al: Active-site inhibitors of mTOR target rapamycin-resistant outputs of mTORC1 and mTORC2. PLoS Biol 7: e38, 2009.

12. Yu K, Shi C, Toral-Barza L, et al: Beyond rapalog therapy: preclinical pharmacology and antitumor activity of WYE-125132 an ATP-competitive and specific inhibitor of mTORC1 and mTORC2. Cancer Res 70: 621-631, 2010.

13. Thoreen CC, Kang SA, Chang JW, et al: An ATP-competitive mammalian target of rapamycin inhibitor reveals rapamycinresistant functions of mTORC1. J Biol Chem 284: 8023-8032, 2009.

14. Janes MR, Limon JJ, So L, et al: Effective and selective targeting of leukemia cells using a TORC1/2 kinase inhibitor. Nat Med 16 205-213, 2010.

15. Schenone S, Brullo C, Musumeci F, et al: ATP-competitive inhibitors of mTOR: an update. Curr Med Chem 18: 2995-3014, 2011.

16. Shao H, Gao C, Tang H, et al: Dual targeting of mTORC1/C2 complexes enhances histone deacetylase inhibitor-mediated anti-tumor efficacy in primary HCC cancer in vitro and in vivo. J Hepatol 56: 176-183, 2012.

17. Zhang YJ, Duan Y and Zheng XF: Targeting the mTOR kinase domain: the second generation of mTOR inhibitors. Drug Discov Today 16: 325-331, 2011.

18. Bai X, Ma D, Liu A, et al: Rheb activates mTOR by antagonizing its endogenous inhibitor, FKBP38. Science 318: 977-980, 2007.

19. Li H, Lin J, Wang X, et al: Targeting of mTORC2 prevents cell migration and promotes apoptosis in breast cancer. Breast Cancer Res Treat 134: 1057-1066, 2012.

20. Chou TC and Talalay P: Quantitative analysis of dose-effect relationships: the combined effects of multiple drugs or enzyme inhibitors. Adv Enzyme Regul 22: 27-55, 1984.

21. Li DM and Feng YM: Signaling mechanism of cell adhesion molecules in breast cancer metastasis: potential therapeutic targets. Breast Cancer Res Treat 128: 7-21, 2011.
22. Song G, Ouyang G and Bao S: The activation of Akt/PKB signaling pathway and cell survival. J Cell Mol Med 9: 59-71, 2005.

23. Cohen SM and Lippard SJ: Cisplatin: from DNA damage to cancer chemotherapy. Prog Nucleic Acid Res Mol Biol 67: 93-130, 2001.

24. Thoreen CC and Sabatini DM: Rapamycin inhibits mTORC1, but not completely. Autophagy 5: 725-726, 2009.

25. Carew JS, Kelly KR and Nawrocki ST: Mechanisms of mTOR inhibitor resistance in cancer therapy. Target Oncol 6: 17-27, 2011.

26. Masri J, Bernath A, Martin J, et al: mTORC2 activity is elevated in gliomas and promotes growth and cell motility via overexpression of rictor. Cancer Res 67: 11712-11720, 2007.

27. Guertin DA, Stevens DM, Saitoh M, et al: mTOR complex 2 is required for the development of prostate cancer induced by Pten loss in mice. Cancer Cell 15: 148-159, 2009.

28. Shorning BY, Griffiths D and Clarke AR: Lkb1 and Pten synergise to suppress mTOR-mediated tumorigenesis and epithelial-mesenchymal transition in the mouse bladder. PLoS One 6: e16209, 2011.

29. Gulhati P, Bowen KA, Liu J, et al: mTORC1 and mTORC2 regulate EMT, motility, and metastasis of colorectal cancer via RhoA and Rac1 signaling pathways. Cancer Res 71: 3246-3256, 2011.

30. Inoki $\mathrm{K}$, Mori $\mathrm{H}$, Wang $\mathrm{J}$, et al: $\mathrm{mTORC} 1$ activation in podocytes is a critical step in the development of diabetic nephropathy in mice. J Clin Invest 121: 2181-2196, 2011.

31. Proud CG: mTOR signalling in health and disease. Biochem Soc Trans 39: 431-436, 2011.

32. Sparks CA and Guertin DA: Targeting mTOR: prospects for mTOR complex 2 inhibitors in cancer therapy. Oncogene 29: 3733-3744, 2010 